\section{Evaluation of Gene Expression of TGF-B and FOXP3 in Individuals Chronically Infected with Trypanosoma Cruzi}

\begin{abstract}
Amanda Vasconcelos do Nascimento ${ }^{1}$, Adriene Siqueira de Melo', Maria da Glória Aureliano Cavalcanti Melo ${ }^{2,3}$, Sílvia Martins Marinho ${ }^{2,3}$, Wilson de Oliveira Júnior ${ }^{2,3}$, Virginia Maria Barros de Lorena ${ }^{1,3 *}$ and Yara de Miranda Gomes ${ }^{1,3}$
\end{abstract}

${ }^{1}$ Departamento de Imunologia, Centro de Pesquisas Aggeu MagalhãesCpqAM, Fiocruz, Recife, Brazil

${ }^{2}$ Ambulatório de Doença de Chagas e Insuficiência Cardiaca, do Hospital Universitário de Cardiologia/PROCAPE, da Universidade de Pernambuco/ UPE, Recife, Brazil

${ }^{3}$ Programa Integrado de Doença de Chagas, Fiocruz, Rio de Janeiro, Brazil

\begin{abstract}
Chagas diseases till poses a serious endemic problem despite the fact that it was discovered over 100 years ago. The mechanisms that lead to the development of clinical manifestations may be attributed to the host immune response against the parasite. Individuals with the in Determinate (IND) form have regulatory mechanisms that limit pathology development. The aim of the present study was to assess the gene expression of TGF- $\beta$, a cytokine with an immuno regulatory profile, and FOXP3, a transcription factor, in patients with chronic Chagas disease after in vitro stimulation of Peripheral Blood Mononuclear Cells (PBMCs) with the recombinant antigens Cytoplasmatic Repetitive Antigen (CRA) and the Flagellar Repetitive Antigen (FRA) from Trypanosoma cruzi. We selected 37 patients with Chagas disease in the Clinics of Chagas Disease and Heart Failure at the Heart Emergency Unit of Pernambuco (Pronto Socorro Cardiológico de Pernambuco-PROCAPE) at the University of Pernambuco (Universidade de Pernambuco-UPE), Recife-PE, Brazil. The
\end{abstract}

*Corresponding author: Virginia Maria Barros de Lorena, Departamento de Imunologia, Centro de Pesquisas Aggeu Magalhães-CPqAM, Fundação Oswaldo Cruz/Fiocruz, Cidade Universitária, Av. Moraes Rego s/n, CEP 50670-420, Recife, Brazil, Tel: +55 8121012563; Fax: +55 8121012640; E-mail lorena@cpqam.fiocruz.br

Citation: do Nascimento AV, de Melo AS, da Glória Aureliano Cavalcant Melo M, Marinho SM, de Oliveira Júnior W, et al. (2016) Evaluation of Gene Expression of TGF-B and FOXP3 in Individuals Chronically Infected with Trypanosoma Cruzi. J Infect Non Infect Dis 2: 012.

Received: September 17, 2015; Accepted: February 17, 2016; Published: March 02, 2016

\begin{abstract}
patients were divided into 3 groups according to clinical aspects of Chagas' disease, the mild Cardiac group (CARD1) $(n=10)$, severe cardiac (CARD2) $(n=10)$ and IND $(n=17)$. PBMCs were cultured in the presence of CRA or FRA and TGF- $\beta$ and FOXP3 gene expression was analyzed through messenger RNA detect on using Real-Time PCR. Although significant differences in gene expression were not observed among the groups of patients, a high production of TGF- $\beta$ was found, where $70 \%$ and $66.16 \%$ of IND individuals were classified as high TGF- $\beta$ producers after stimulation with CRA and FRA, respectively. This result suggests that this cytokine may be involved in the immune modulatory response, controlling morbidity in this group of patients. Thus, we believe that further studies with other molecules that characterize a regulatory response along with TGF- $\beta$ and FOXP3, such as CD103, CD122 and CD152 (CTLA-4), may contribute to a better prognosis of patients with chronic Chagas disease.
\end{abstract}

\section{Abbreviations}

$\begin{array}{llll}\text { CARD1 } & : & \text { Mild Cardiac Alterations } \\ \text { CARD2 } & : & \text { Severe Cardiac Alterations } \\ \text { IND } & : & \text { Asymptomatic, Indeterminate form } \\ \text { CRA } & : & \text { Cytoplasmatic Repetitive Antigen } \\ \text { NI } & : & \text { Non-infected } \\ \text { FRA } & : & \text { Flagellar Repetitive Antigen } \\ \text { PBMCs } & : & \text { Peripheral Blood Mononuclear Cells }\end{array}$

\section{Introduction}

Chagas disease is caused by the protozoan Trypanosoma cruzi and it is a major public health problem, affecting millions of people in Central and South Americas. There are an estimated 9.8 million people infected with T. cruzi throughout the world, mainly in Latin America [1]. In Brazil, approximately three million people are infected [2]. In humans, the disease is characterized by an acute phase with high parasitemia levels, followed by a chronic, continuous phase, when detection of circulating parasites through direct blood tests is difficult.

Most infected people develop the chronic phase and either remain with the asymptomatic form (also called indeterminate-IND) throughout their lives, or progress to symptomatic clinical forms after 10 to 20 years of infection. Approximately $30 \%$ of patients in the chronic phase develop the cardiac form (CARD) of the disease. This form has a wide range of symptoms that may be manifested as mild (CARD1) or severe (CARD2) cardiac alterations $[4,5]$.

Several studies on Chagas diseases how that although individuals who develop the CARD form are able to control parasitemia, they may not be able to mount an efficient immune regulatory response and may present an exacerbated inflammatory response, leading to tissue damage. Conversely, individuals that remain asymptomatic may be able to reduce the number of parasites in the early stage of infection through regulatory mechanisms that limit pathology development [6,7].

The immune mechanisms by which individuals with the IND form start to present heart-associated clinical manifestations after 10-20 years of infection have not been fully elucidated [8]. One of 
these mechanisms may be through the activity of regulatory $\mathrm{T}$ cells, characterized phenol typically in humans as CD4+ CD25 High FOXP3+ T cells [9]. These cells are an important source of regulation of the immune response, controlling the local inflammatory response and avoiding severe tissue destruction [10]. According to Araujo et al., [11], patients presenting the CARD form have specific populations of CD8+ T cells, which results in the production of inflammatory cytokines. These cytokines may be involved in the establishment of an exacerbated inflammatory process, leading to an uncontrolled immune response. The latter is characterized by the absence or in ability of action of regulatory $\mathrm{T}$ cells (Treg cells) through the production of their main cytokine, TGF- $\beta$, which modulates the expression of FOXP3 by Tregs and is also able to transform peripheral CD4+ CD25 - T cells in CD4+ CD25+ cells [12]. On the contrary, patients with the IND form have an effective, possibly transient, immune regulatory response that controls the response to infection mediated by regulatory effector $\mathrm{T}$ cells [11].

Several studies have used specific, specific antigens of T. cruzi, known as Cytoplasmatic Repetitive Antigen (CRA) and Flagellar Repetitive Antigen (FRA) [13,14] with aim to help clarifying the immune pathology of Chagas disease [15]. The role of CRA and FRA in the immune response of patients with chronic Chagas disease has been studied by our research group and promising results have been generated [16-18]. Here, the immune response of patients with chronic Chagas disease was assessed after in vitro stimulation of Peripheral Blood Mononuclear Cells (PBMCs) with CRA and FRA.

The present study aimed to describe the gene expression profiles of TGF- $\beta$ and FOXP 3 in patients with chronic Chagas disease after in vitro stimulation with the recombinant $T$. cruzi antigens CRA and FRA. The results obtained here may contribute to a better understanding of the development of symptomatic forms of the disease.

\section{Material and Methods}

\section{Study population}

Individuals with Chagas disease were selected in the Clinics of Chagas Disease and Heart Failure at the Heart Emergency Unit of Pernambuco (Pronto Socorro Cardiológico de Pernambuco-PROCAPE) at the University of Pernambuco (Universidade de Pernambuco-UPE), Recife-PE, Brazil. The inclusion criteria were three: 1) positive serology for Chagas infection in two tests with different methodology principles (one test using recombinant antigens and the other test using crude antigens of T. cruzi);2) patients that have undergone clinical exams to characterize the clinical form (physical examination, electrocardiogram, echocardiogram, and chest and esophagus X-rays), and 3) patients that have not under gone etiological treatment. The CARD group was further divided into CARD1 $(\mathrm{n}=10,50-74$ years old; 4 women and 6 men), which included patients without cardiac dilation and with an ejection fraction $>55 \%$ on the echocardiogram, and CARD2 $(n=10,43-73$ years old; 4 women and 6 men), which included patients with clinical and or echo cardio graphic and radiological signs of fast heart rate and an ejection fraction $<40 \%$ on the echocardiogram. Patients with the Indeterminate form (IND) $(\mathrm{n}=17,32-68$ years old; 9 women and 8 men) were selected by not having any heart and digestive alterations, but with positive serology for T. cruzi infection. A group of Non-Infected (NI) volunteers $(n=7)$ was included for comparison with individuals with Chagas disease by using the following criteria: 1) people that have in habited an endemic Chagas disease area; 2) people that have never received blood transfusion, and
3) people with non-reactive serologic tests for Chagas disease. The individuals included in the study signed an Informed Consent Form (ICF), which was previously approved by the Ethics Committee of the Centro de Pesquisas Aggeu Magalhães-Fiocruz (CPqAM/Fiocruz) under the ID No.03/2009.

\section{PBMCs culture and stimulation with recombinant T.cruzi antigens}

PBMCs were obtained from samples collected in tubes containing sodium heparin through Ficoll-Paque density centrifugation (GE Healthcare, Uppsala, Sweden), as previously described [19]. The cell culture was performed as described by Melo et al., [20]. CRA and FRA antigens were obtained as described by Krieger et al., [14]. They were then analyzed by electrophoresis in a poly acryl amide gel in the presence of sodium dodecyl sulfate [21] and by silver staining [22] to assess Escherichia coli contamination.

\section{Total RNA extraction, reverse transcription reaction and real-time PCR}

Total RNA was isolated from PBMCs using Trizol reagent (Invitrogen ${ }^{\mathrm{Tw}}$, Carlsbad, CA, USA) according to the manufacturer's instructions. Next, total RNA was incubated with deoxyribonuclease I using the Amplification Kit Grade (Invitrogen ${ }^{\mathrm{TM}}$ ) at a final RNA concentration of $100 \mathrm{ng} / \boldsymbol{\mu L}$ in order to ensure complete elimination of genomic DNA in the samples. Reverse Transcription (RT) to obtain cDNA was performed using the High Capacity cDNA Reverse Transcription with RNAse Inhibitor kit (Applied Biosytems ${ }^{\mathrm{RM}}$, Foster City, CA, USA), according to manufacturer's instructions. The cDNA was then used in Real-Time PCR assays, as described by Melo et al., [20]. The sequences of primers and probes (200nM) used to amplify $\beta$-actin (endogenous gene) and TGF- $\beta$ genes were described by Mocellin et al., [23] and Mwacharo et al., [24], respectively, and were: $\beta$-Actin sense-5'-GGCACCCAGCACAATGAAG-3' and anti-sense-5'-GCCGATCCACACGGAGTACT-3, probe-VIC TCAAGATCATTGCTCCTCCTGAGAGCGC-MGB; TGF- $\beta$ sense-5'-CGAGAAGCGGTACCTGAAC-3' and anti-sense-5'-TGAGGTATCGCCAGGAATTGT-3', probe-6FAM-CAGCACGTGGAGCTGTACCAGAAATACAGC-MGB. Primers and probes for the FOXP3 gene were obtained directly from the custom primers and probes by manufacturer (Applied Bio systems ${ }^{\circledR}$-Product Number: 4304971). Standard curves of the targets showed similar efficiency $(>99 \%)$. The relative quantitation was calculated according by Wilhelm et al. [25]. The relative quantification was established by the ratio between the mean CT (cycle threshold) values of the target gene and the reference gene ( $\beta$-actin) in each stimulated sample / reference sample (negative controls). In addition, the efficiency correction was performed according by Pfaffl [26].

\section{Statistical analysis}

A descriptive analysis was performed to expose the obtained results. To test data homogeneity assumption the Levene test was used and homogeneity was confirmed by the Barlet test. To compare the gene expression mean of TGF- $\beta$ cytokine and FOXP3 among the groups and for each stimulus, ANOVA followed by Tukey test were used. To asses "high" and "low" producers of the cytokine and FOXP3, the chi-square test of proportions was applied. All conclusions were based on a significance level of 5\%. Excel 2000 was used to build the database and Graph Pad Prism 5.0 was used to perform the statistical tests. 
Citation: do Nascimento AV, de Melo AS, da Glória Aureliano Cavalcanti Melo M, Marinho SM, de Oliveira Júnior W, et al. (2016) Evaluation of Gene Expression of TGF-B and FOXP3 in Individuals Chronically Infected with Trypanosoma Cruzi. J Infect Non Infect Dis 2: 012.

- Page 3 of $5 \cdot$

\begin{tabular}{|c|c|c|c|c|c|c|c|c|c|c|}
\hline \multicolumn{5}{|c|}{ Median Expression TGF- $\beta$ and FOXP3 (+/-Standard Deviation) } & \multicolumn{6}{|c|}{ Frequency of low and high gene expression TGF- $\beta$ and FOXP3 (\%) } \\
\hline & IND $n=17$ & CARD1 $n=10$ & CARD2 $n=10$ & $\mathrm{NI} n=7$ & & Cut-off & IND $n=17$ & CARD1 $n=10$ & CARD2 $n=10$ & $\mathrm{NI} n=7$ \\
\hline CRA TGF- $\beta$ & $1,14(0,73-1,89)$ & $0,95(0,63-1,31)$ & $0,95(0,39-1,17)$ & $1,13(0,75-1,71)$ & CRA TGF- $\beta$ & 1,06 & 70 & 30 & 50 & 57,14 \\
\hline FRA TGF- $\beta$ & $1,01(0,71-1,35)$ & $0,92(0,72-1,37)$ & $0,84(0,28-1,30)$ & $1,06(0,63-1,35)$ & FRA TGF- $\beta$ & 0.96 & 63,16 & 40 & 40 & 72,43 \\
\hline CRAFOXP3 & $1,25(0,63-5,94)$ & $0,94(0,87-1,09)$ & $2,29(0,32-12,8)$ & $0,97(0,91-1,06)$ & CRA FOXP3 & 1,08 & 21,05 & 10 & 33,33 & 0 \\
\hline FRA FOXP3 & $1,02(0,32-2,92)$ & $0,97(0,87-1,15)$ & $1,17(0,34-2,31)$ & $0,94(0,67-1,06$ & FRA FOXP3 & 1,03 & 21,05 & 20 & 33,33 & 12,5 \\
\hline
\end{tabular}

Table 1: Detection of TGF- $\beta$ (A) and FOXP3 transcription factor (B) gene expression in PBMCs of patients with chronic Chagas disease and in non-infected individuals after stimulation with the Cytoplasmic Repetitive Antigen (CRA) and Flagellar Repetitive Antigen (FRA) of T. cruzi.

Note: Expression of these genes are shown in patients with the clinical Cardiac form without Dilatation (CARD1) $(n=10)$, patients with the clinical Cardiac form with Dilatation (CARD2 (gene expression of TGF- $\beta(n=10)$ and gene expression of FOXP3 $(n=9)$ ), patients with the Indeterminate form (IND) $(n=17)$, and Non-Infected (NI) individuals $(n=7)$.

\section{Results}

\section{Gene expression of TGF- $\beta$ cytokine and FOXP3 transcrip- tion factor after in vitro stimulation with recombinant $T$. cruzi antigens.}

Analysis of the differences in gene expression levels of TGF- $\beta$ and FOXP3 was performed after in vitro stimulation of cells with T. cruzi recombinant antigens (Rec-Ags) CRA and FRA in comparison to cultures without stimulation, where no statistically significant differences were found for the two genes in any group. The results of the ANOVA test for CRA (TGF- $\beta(\mathrm{p}=0.13)$ and FOXP3 $(\mathrm{p}=0.38)$ ) and FRA (TGF- $\beta(\mathrm{p}=0.13)$ and FOXP3 $(\mathrm{p}=0.67))$ antigens showed no difference between groups of individuals were found (Table 1).

Frequency of high-and low-producers of the transcription factor FOXP3after in vitro stimulation with the recombinant $T$. cruzi antigens CRA and FRA.

In order to improve the detection of gene expression changes of TGF- $\beta$ cytokine and FOXP 3 transcription factor, a cut-off value was set corresponding to the global average expression values from all individuals (IND, CARD1, CARD2 and NI). The individual values of each group were inserted above or below the cut-off value and used to investigate the frequency of cytokine expression among groups. Although there was no statistically significant difference in gene expression, it was observed that most patients with the IND form (70\%) were classified as "high-producers" of TGF- $\beta$, when compared CARD 1 (30\%), CARD 2 (50\%) and NI (57.14\%) individuals after stimulation with CRA. When we used the FRA, we found that $63.16 \%$ of individuals in the IND form were above the cut-off, compared to CARD1 (40\%), CARD2 (40\%) and NI (72.43\%) (Table 1).

As for FOXP3 gene, we found that a small proportion of individuals from all groups. After stimulation with CRA: IND (21.05\%), CARD1 (10\%), CARD2 (33.33\%) and NI (0\%). When we the FRA: IND (21.05\%), CARD1 (20\%), CARD2 (33.33\%) and NI (12.5\%). No statistically significant difference was observed for FOXP3 gene with both Rec-Ags used (Table 1).

\section{Discussion}

Few studies have investigated the role of soluble factors involved in the immune response against Chagas disease. Similar to the results reported here, studies such as Rezende-oliveira et al., [27] in which TGF- $\beta$ levels were measured in the supernatant of mononuclear cells infected with T. cruzi, and Nougueira et al., [28], who analyzed TGF- $\beta$ gene expression through real time PCR in patients with chronic Chagas disease, reported no statistically significant differences in TGF- $\beta$ expression. This allows us to conclude that TGF- $\beta$ synthesis assessed through qPCR after cultivation with CRA and FRA Rec-Ags may not be used as a prognostic marker of the evolution of the clinical forms of chronic Chagas disease.

Thus, despite the fact that a few studies suggest an association of the IND form with a high production of anti-inflammatory and regulatory cytokines such as IL- 4 and IL-10 and TGF- $\beta$, and of the CARD form with high levels of pro-inflammatory cytokines such as IFN- $\gamma$ and TNF- $\alpha[29,30]$ ) here we found results similar to other studies, where no relationship was observed between the production profile of a certain cytokine and Chagas disease clinical symptoms $[17,20,31]$.

The FOXP3 gene, unlike other genes associated to regulatory $\mathrm{T}$ cells (Tregs), is specific of the Treg lineage [33]. In mice, the ectopic expression of FOXP3 in naive T cells is able to convert them into Tregs with similar phenotype and function of innate Tregs, conferring them an in vivo and in vitro suppress or activity and increased expression of molecules characteristic of Tregs such as CD25+, CTLA-4 (T Lymphocyte-Associated Protein 4) and GITR (Glucocorticoid-Induced TNRF-Related Protein) [34]. In humans, it is still unclear whether FOXP 3 is a specific marker for Tregs, as activated T cells may transiently express FOXP3 (even if at low levels when compared with T CD4+ CD25 high) [35]. Our results are consistent with data obtained previously by our group. The frequency of FOXP3 molecule in $\mathrm{CD} 4+\mathrm{CD} 25+\mathrm{T}$ cells was evaluated among individuals with different clinical forms of Chagas disease through flow cytometry. No statistical difference was observed among groups in the presence of CRA or FRA, despite the fact that $75 \%$ and $70 \%$ of IND individuals expressed FOXP3 after stimulation with CRA and FRA, respectively [36]. Thus, the expression of FOXP 3 under the conditions studied here may not be used as a prognostic marker for the evolution of chronic clinical forms of Chagas disease.

Various factors such as, for example, the strain of the parasite, time of infection and reinfection potential may be important in the progression of Chagas disease. The fact that the experimental groups were made up of individuals from the same endemic area of the country and the median age between the groups were similar, we believe that our sample has undergone minimal interference by these factors. However, we know that the genetic background and consequently the development of the immune response of the host during infection is a limiting factor in any immunological study.

The categorization of patients in regard to the production of cytokines has been used to assess individuals that within a certain group exhibit high production levels of cytokines [37,38]). Gomes et al., [37], when studying IFN- $\gamma$ production, divided the groups of patients with cardiac changes according to disease severity. In this way, it was possible to establish a correlation between "high" and "low" 
Citation: do Nascimento AV, de Melo AS, da Glória Aureliano Cavalcanti Melo M, Marinho SM, de Oliveira Júnior W, et al. (2016) Evaluation of Gene Expression of TGF-B and FOXP3 in Individuals Chronically Infected with Trypanosoma Cruzi. J Infect Non Infect Dis 2: 012.

IFN- $\gamma$ producers and the clinical status of the patient. Lorena et al., Melo et al., and Vitelli-Avellar et al., [16,20,38], established a cut-off value based on the overall mean among all study subjects and were able to separate them into "high" and "low" cytokine producers.

In the present study, we evaluated the gene expression patterns of TGF- $\beta$ cytokine by establishing a cut-off value and consequently identifying high expression levels. Although we did not observe statistically significant differences, we found an increased production of TGF- $\beta$ mostly in individuals with the IND form, with frequencies of $70 \%$ and $63.16 \%$ after stimulation with CRA and FRA, respectively.

The fact that most IND individuals are TGF- $\beta$ producers suggests that this cytokine may play a role in the immunomodulatory response, controlling morbidity in this group of patients, as previously described in the literature [12]. However, further evaluation of cells producing this cytokine through flow cytometry (cell phenol typing) may help to clarify the possible role of a Th1-type inflammatory response by these cells in controlling the disease.

Detection of FOXP3 in regulatory T cells has been the subject of intense study aimed to elucidate the immune regulatory mechanism of these cells in Chagas disease [11,39]. In the present work, when analysis of patient distribution was performed, no difference was observed among the groups IND, CARD1, CARD2 and NI when PBMCs were stimulated in vitro with the Rec-Ags CRA and FRA. However, it would be interesting to assess the association of FOXP3 expression data with other regulatory $\mathrm{T}$ cells markers such as CD103, CD122 and CD152 (CTLA-4).

\section{Conclusion}

We believe that the immunological mechanisms involved, in the joint interaction of immune cells, and the differential genetic susceptibility of the host, lead to a highly complex pathology, imposing difficulties in the detection of a specific immune response. Thus, further follow-up studies of these individuals are necessary to help clarifying the immune pathological mechanisms behind Chagas disease and to offer data to support the development of vaccines and efficient immune therapies against this disease.

\section{Acknowledgment}

The authors are grateful to Dr. Cristina Tavares and Marisa Melo from PROCAPEUPE for selecting the patients included in this study and to Mineo Nakazawa at CPqAM/Fiocruz for technical assistance. The authors also thank the Program for Technological Development in Tools for Health-PDTIS-FIOCRUZ for use of its facilities. This research study received financial support from the "Conselho Nacional de Desenvolvimento Científico e Tecnológico" (CNPq) (Edital Universal No. 478572/2009-3). YM Gomes is a CNP q research fellow (Fellowship No. 304896/2009-8). During the study, AV Nascimen to received a Scientific Initiation fellowship from $\mathrm{CNPq}$, VMB Lorena received a Ph.D. fellowship from CNPq, and AS Melo received a M.Sc. fellowship from the "Coordenação de A perfeiçoamento de Pessoal de Nível Superior” (CAPES).

\section{Reference}

1. World Health Organization (2012) Research priorities for Chagas disease, human African trypanosomiasis and leishmaniasis. World Health Organ Tech Rep Ser v-xii, 1-100.

2. Petherick $A(2010)$ Chagas disease in the Chaco. Nature 465: 18-20.
3. Reis MM, Higuchi ML, Benveneti LA, Aiello VD, Gutierrez PS, et al. (1997) An in situ quantitative immune histochemical study of cytokines and IL-2R+ in chronic human chagasic myocarditis: correlation with the presence of myocardial Trypanosoma cruzi antigens. Clin Immunol Immuno pathol 83: 165172.

4. Ribeiro ALP, Rocha MOC (1998) Forma indeterminada da doença de Chagas: considerações acerca do diagnóstico e do prognóstico. Revista da Sociedade Brasileira de Medicina Tropical 31: 301-314.

5. Rocha MO, Teixeira MM, Ribeiro AL (2007) An update on the management of Chagas cardiomyopathy. Expert Rev Anti Infect Ther 5: 727-743.

6. Dutra WO, Gollob KJ (2008) Current concepts in immunoregulation and pathology of human Chagas disease. Curr Opin Infect Dis 21: 287-292.

7. Dutra WO, Menezes CAS, Villani FNA, Costa GC, Silveira ABM, et al. (2009) Cellular and genetic mechanisms involved in the generation of protective and pathogenic immune responses in human Chagas disease. Memórias do Instituto Oswaldo Cruz 104: 208-218.

8. Gutierrez FR, Guedes PM, Gazzinelli RT, Silva JS (2009) The role of parasite persistence in pathogenesis of Chagas heart disease. Parasite Immunol 31: 673-685.

9. Baecher-Allan C, Viglietta V, Hafler DA (2004) Human CD4+CD25+ regulatory T cells. Semin Immunol 16: 89-98.

10. Cools N, Ponsaerts P, van Tendeloo VF, Berneman ZM (2007) Balancing between immunity and tolerance: an inter play between dendritic cells regulatory T cells, and effector T cells. J Leukoc Biol 82: 1365-1374.

11. De Araújo FF, da Silveira AB, Correa-Oliveira R, Chaves AT, Adad SJ, et al. (2011) Characterization of the presence of Foxp3+ T cells from patients with different clinical forms of Chagas' disease. Human Pathology 42: 299-301.

12. Chen W, Jin W, Hardegen N, Lei KJ, Li L, et al. (2003) Conversion of peripheral CD4+CD25- naive T cells to CD4+CD25+ regulatory T cells by TGF-beta induction of transcription factor Foxp3. J Exp Med 198: 1875-1886.

13. Lafaille JJ, Linss J, Krieger MA, Souto-Padrón T, de Souza W, et al. (1989) Structure and expression of two Trypanosoma cruzigenes encoding antigenic proteins bearing repetitive epitopes. Molecular and Biochemical Parasitology 35: 127-136.

14. Krieger MA, Almeida E, Oelemann W, Lafaille JJ, Pereira JB, et al. (1992) Use of recombinant antigens for the accurate immunodiagnosis of Chagas' disease. Am J Trop Med Hyg 46: 427-434.

15. Lorca M, Gonzales A, Veloso C, Reyes V, Vergata U (1992) Immunodetection of antibodies in sera from symptomatic and asymptomatic chilean Chagas' disease patients with Trypanosoma cruzi recombinant antigens. The American Journal of Tropical Medicine and Hygiene 46: 44-49.

16. Lorena VM, Lorena IM, Braz SC, Melo AS, Melo MF, et al. (2010) Cytokine levels in serious cardiopathy of Chagas disease after in vitro stimulation with recombinant antigens from Trypanosoma cruzi. Scand J Immunol 72: 529539.

17. Vasconcelos RH, Amaral FN, Cavalcanti MG, Silva ED, Ferreira AG, et al. (2010) Increased levels of IgA antibodies against CRA and FRA recombinant antigens of Trypanosoma cruzi differentiate digestive forms of Chagas disease. Hum Immunol 71: 964-967.

18. Verçosa AF, Lorena VM, Carvalho CL, Melo MF, Cavalcanti MG, et al. (2007) Chagas' disease: IgG isotypes against Cytoplasmic (CRA) and Flagellar (FRA) recombinant repetitive antigens of Trypanosoma cruzi in chronic Chagasic patients. J Clin Lab Anal 21: 271-276.

19. Lorena VM, Verçosa AF, Machado RC, Moitinho-Silva L, Cavalcanti MG, et al. (2008) Cellular immune response from Chagasic patients to CRA or FRA recombinant antigens of Trypanosoma cruzi. J Clin Lab Anal 22: 91-98.

20. de Melo AS, de Lorena VM, de Moura Braz SC, Docena C, de Miranda Gomes $Y(2012)$ IL-10 and IFN-c gene expression in chronic Chagas disease patients after in vitro stimulation with recombinant antigens of Trypanosoma cruzi. Cytokine 58: 207-212. 
21. Laemmli UK (1970) Cleavage of structural proteins during the assembly of the head of bacteriophage T4. Nature 227: 680-685.

22. Morrissey JH (1981) Silver stain for proteins in poly acryl amide gel: a modified procedure with enhanced uniform sensitivity. Analytical Bio chemistry 117: $307-310$.

23. Mocellin S, Provenzano M, Rossi CR, Pilati P, Nitti D, et al. (2003) Use of quantitative real-time PCR to determine immune cell density and cytokine gene profile in the tumor microenvironment. J Immunol Methods 280: 1-11.

24. Mwacharo J, Dunachie SJ, Kai O, Hill AV, Bejon P, et al. (2009) Quantitative $\mathrm{PCR}$ evaluation of cellular immune responses in Kenyan children vaccinated with a candidate malaria vaccine. PLoS One 4: 8434

25. Wilhelm J, Pingoud A (2003) Real-time polymerase chain reaction. Chembiochem 4: 1120-1128.

26. Pfaffl MW (2001) A new mathematical model for relative quantification in real-time RT-PCR. Nucleic Acids Res 29: 45.

27. Rezende-oliveira K, Sarmento RR, Rodrigues-junior V (2012) Production of cytokine and chemokines by human mononuclear cells and whole blood cells after infection with Trypanosoma cruzi. Revista da Sociedade Brasileira de Medicina Tropical 45: 45-50.

28. Nougueira LG, Santos RHB, Fiorelli Al, Mairena EC, Benvenuti LA, et al (2014) Myocardial Gene Expression of T-bet, GATA-3, Ror-yt, FoxP3 and Hallmark Cytokines in Chronic Chagas Disease Cardiomyopathy: An Essentially Unopposed TH1-Type Response. Mediators of inflammation.

29. Pissetti CW, Correia D, de Oliveira RF, Llaguno MM, Balarin MA, et al. (2011) Genetic and functional role of TNF-alpha in the development Trypanosoma cruzi infection. PLoS Negl Trop Dis 5: 976.

30. Poveda C, Fresno M, Gironès N, Martins-Filho AO, Ramirez JD, et al. (2014) Cytokine Profiling in Chagas Disease: Towards Understanding the Association with Infecting Trypanosoma cruzi Discrete Typing Units (A Benefit Trial Sub-Study). PLoS One 9: 91154.
31. Araujo FF, Gomes JA, Rocha MO, Williams-Blangero S, Pinheiro VM, et al (2007) Potential role of CD4+CD25HIGH regulatory $\mathrm{T}$ cells in morbidity in Chagas disease. Front Biosci 12: 2797-2806.

32. Fontenot JD, Gavin MA, Rudensky AY (2003) Foxp3 programs the develop ment and function of CD4+CD25+ regulatory T cells. Nat Immunol 4: 330336.

33. Khattri R, Cox T, Yasayko SA, Ramsdell F (2003) An essential role for Scurfin in CD4+CD25+ T regulatory cells. Nat Immunol 4: 337-342.

34. Hori S, Nomura T, Sakaguchi S (2003) Control of regulatory T cell development by the transcription factor Foxp3. Science 299: 1057-1061.

35. Gavin M, Rudensky A (2003) Control of immune homeostasis by naturally arising regulatory CD4+ T cells. Curr Opin Immunol 15: 690-696.

36. de Moura Braz SC, de Melo AS, da Glória Aureliano de Melo Cavalcanti M, Martins SM, de Oliveira W Jr, et al. (2014) Increase in the expression of CD4 + CD25+ lymphocytic T cells in the indeterminate clinical form of human Chagas disease after stimulation with recombinant antigens of Trypanosoma cruzi. J Clin Immunol 34: 991-998.

37. Gomes JA, Bahia-Oliveira LM, Rocha MO, Martins-Filho OA, Gazzinelli G, et al. (2003) Evidence that development of severe cardiomyopathy in human Chagas' disease is due to a Th1-specific immune response. Infect Immun 71: 1185-1193.

38. Vitelli-Avelar DM, Sathler-Avelar R, Teixeira-Carvalho A, Pinto Dias JC, Gontijo ED, et al. (2008) Strategy to assess the overall cytokine profile of circulating leukocytes and its association with distinct clinical forms of human Chagas disease. Scand J Immunol 68: 516-525.

39. de Araújo FF, Corrêa-Oliveira R, Rocha MO, Chaves AT, Fiuza JA, et al (2012) Foxp3+CD25(high) CD4+ regulatory T cells from indeterminate patients with Chagas disease can suppress the effector cells and cytokines and reveal altered correlations with disease severity. Immunobiology 217: 768777. 